\title{
Stability of orthotropic plates
}

\author{
Mykola Surianinov ${ }^{1, *}$, Dina Lazarieva ${ }^{1}$, and Iryna Kurhan $^{2}$ \\ ${ }^{1}$ Odesa State Academy of Civil Engineering and Architecture, Odesa, 65029, Ukraine \\ ${ }^{2}$ Odesa National Polytechnical University, Odesa, 65044, Ukraine
}

\begin{abstract}
The solution to the problem of the stability of a rectangular orthotropic plate is described by the numerical-analytical method of boundary elements. As is known, the basis of this method is the analytical construction of the fundamental system of solutions and Green's functions for the differential equation (or their system) for the problem under consideration. To account for certain boundary conditions, or contact conditions between the individual elements of the system, a small system of linear algebraic equations is compiled, which is then solved numerically. It is shown that four combinations of the roots of the characteristic equation corresponding to the differential equation of the problem are possible, which leads to the need to determine sixty-four analytical expressions of fundamental functions. The matrix of fundamental functions, which is the basis of the transcendental stability equation, is very sparse, which significantly improves the stability of numerical operations and ensures high accuracy of the results. An analysis of the numerical results obtained by the author's method shows very good convergence with the results of finite element analysis. For both variants of the boundary conditions, the discrepancy for the corresponding critical loads is almost the same, and increases slightly with increasing critical load. Moreover, this discrepancy does not exceed one percent. It is noted that under both variants of the boundary conditions, the critical loads calculated by the boundary element method are less than in the finite element calculations. The obtained transcendental stability equation allows to determine critical forces both by the static method and by the dynamic one. From this equation it is possible to obtain a spectrum of critical forces for a fixed number of half-waves in the direction of one of the coordinate axes. The proposed approach allows us to obtain a solution to the stability problem of an orthotropic plate under any homogeneous and inhomogeneous boundary conditions.
\end{abstract}

\section{Introduction}

The development level of production at the present stage is characterized by the widespread introduction of new technologies for the manufacture of high-strength materials with orthotropic (orthogonally anisotropic) properties.

Such materials include fiberglass; composite materials reinforced with sequentially alternating layers of fibers in two mutually perpendicular directions; glued wood plates; sheet rolled metals, in which anisotropy begins to appear upon transition to the plastic stage of work, etc.

The widespread use of materials with anisotropic properties has given rise to large-scale studies in the field of mechanics of anisotropic structures and, in the first place, plates.

In many industries, designs in the form of plates made of orthotropic materials with three planes of symmetry of elastic properties are widely used. Under certain conditions, the operation of such plates is accompanied by the appearance of compressive stresses in the median plane, which can lead to a loss of stability and bearing capacity of the plate.

Determining the critical load on a plate presents serious mathematical difficulties not only for orthotropic, but also for isotropic plates. In well-known monographs and reference books, only the stability problem of a rectangular plate with a hinged support along the contour is solved [1-4].

\section{Latest researches analysis}

The stability problem of an isotropic rectangular plate loaded on two opposite edges by forces distributed according to a linear law was first solved by I. G. Bubnov and S. P. Timoshenko [5]. For an orthotropic plate, this problem was solved by S. G. Lehnitsky [6]. All these classical solutions are obtained for the case of the edges simple support of the plate in the form of double trigonometric rows.

From later works, we note I. E. Harik papers [7, 8], the first of which sets out a numerical-analytical method for the analysis of orthotropic rectangular plates subject to uniform, linearly changing and piecewise-continuous plane loads; the decision procedure is based on the

\footnotetext{
* Corresponding author: sng@ogasa.org.ua
} 
classical method of variables separation. And the second one proposes an analytical method for solving the problem of elastic bending of orthotropic rectangular plates under different boundary conditions. A generalization of all the results obtained in this direction was made by F. Bloom and D. Coffin in an interesting Handbook [9], which is, in our opinion, the most comprehensive review of theoretical methods for calculating the stability of thin plates.

There are very few contemporary works devoted to the stability of anisotropic plates. We note the paper [10], where the stability problem of an anisotropic plate is solved (but again with a hinged support along the contour) and the work [11], where the stability problem is solved for pure bending of an orthotropic plate, in which two opposite edges are free and two other edges articulated. The finite difference method was used here. The works of foreign authors are based primarily on the use of numerical methods, and, most often, the finite element method and its modifications are encountered. The paper [12] considers the analysis of vibrations and stability of thick orthotropic plates using finite elements based on the Treffz hybrid formula. The Treffz type finite element method (TFEM) is used by C. Young [13] to solve some potential problems in orthotropic environment. The method of boundary elements in classical form was used in [14]. Here, fundamental solutions are obtained for orthotropic thick plates with allowance for lateral shear strain. Boundary integral equations are formulated that are adapted to arbitrary boundary conditions. Examples of numerical implementation are given.

\section{Research aim}

The aim of research is an experimental study of the influence of steel fiber on the bearing capacity, deformability and crack resistance of serial reinforced concrete multi-hollow slabs manufactured in the factory.

\section{Materials and methods}

Problems of this kind can be solved by numerical methods, such as the finite element method, the finite difference method, the R-function method, etc., but it is advisable to verify the results by any analytical method. It seems that such is the NA BEM. As is known [15], the basis of this method is the analytical construction of the fundamental system of solutions and Green's functions for the differential equation (or their system) for the problem under consideration. To account for certain boundary conditions, or contact conditions between the individual elements of the system, a small system of linear algebraic equations is compiled, which is then solved numerically. In this case, no restrictions are imposed either on the boundary conditions or on the nature of the external load. Note that the method is strictly mathematically justified, since it uses fundamental solutions of differential equations, therefore, taking into account the initially accepted hypotheses, it allows to obtain exact values of the desired quantities of the problem.

\section{Research results}

The differential stability equation of an orthotropic plate within the framework of the Kirchhoff-Love hypothesis (Fig. 1) can be written as

$$
\begin{aligned}
& D_{1} \frac{\partial^{4} W(x, y)}{\partial x^{4}}+2 D_{3} \frac{\partial^{4} W(x, y)}{\partial x^{2} \partial y^{2}}+D_{2} \frac{\partial^{4} W(x, y)}{\partial y^{4}}+ \\
& +N_{x}(y) \frac{\partial^{2} W(x, y)}{\partial x^{2}}+2 N_{x y} \frac{\partial^{2} W(x, y)}{\partial x \partial y}+ \\
& +N_{y}(x) \frac{\partial^{2} W(x, y)}{\partial y^{2}}=q(x, y)
\end{aligned}
$$

where stiffnesses are defined by formulas

$$
\begin{aligned}
& D_{1}=\frac{E_{x} h^{3}}{12\left(1-\mu_{x y} \mu_{y x}\right)} ; \quad D_{2}=\frac{E_{y} h^{3}}{12\left(1-\mu_{x y} \mu_{y x}\right)} ; \\
& D_{3}=D_{1} \mu_{x y}+2 D_{k}=D_{2} \mu_{y x}+2 D_{k} ; \quad D_{k}=\frac{G h^{3}}{12} .
\end{aligned}
$$

Here $E_{x}, E_{y}-$ moduli of elasticity in the axis directions; $G$ - shear modulus; $h$ - plate thickness; $\mu_{x y}$, $\mu_{y x}$ - Poisson's ratio; $W(x, y)$ - deflection amplitude value; $N_{x}(y) ; N_{x y} ; N_{y}(x)$ - forces in the middle plane; $q(x, y)$ - transverse load amplitude.

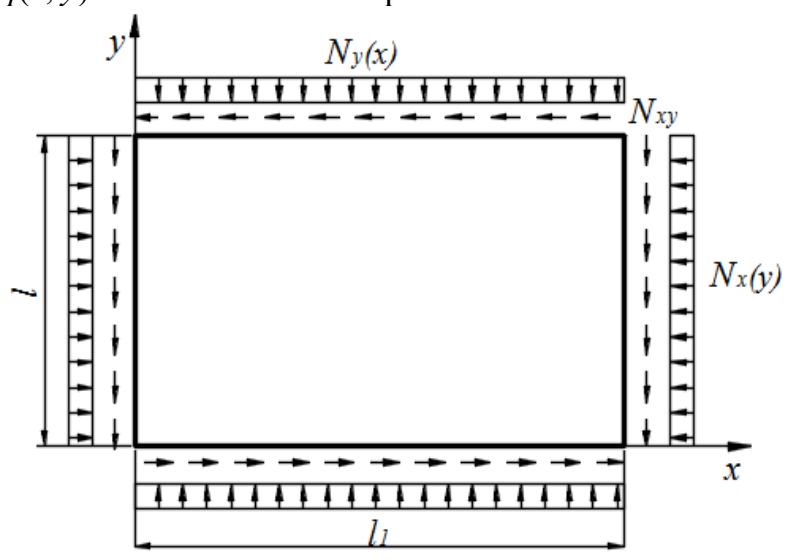

Fig. 1. Plate loads.

The stability equation (1) is of the fourth order and is a partial differential equation. Function $W(x, y)$, which is the solution of this equation, depends on two variables. The transition from a two-dimensional problem to a onedimensional one, as required by the algorithm of the NA BEM, can be accomplished by applying the Kantorovich-Vlasov variational method.

Let's expand the deflection $W(x, y)$ into functional series:

$$
\begin{aligned}
& W(x, y)=W_{1}(y) X_{1}(x)+W_{2}(y) X_{2}(x)+\ldots \\
& \ldots+W_{n}(y) X_{n}(x) .
\end{aligned}
$$

Functions $X_{i}(x)$ it is necessary to choose such that they most accurately describe the shape of the curved 
surface of the plate in the direction of the axis $o x$. Deflection curves for a beam that has the same abutment conditions as the plate in the direction of the axis $o x$ meet his requirement. To select the lateral deflection distribution function $X_{i}(x)$ there are known two methods - static and dynamic [15]. When using the static method, the deflection of the beam is determined by the static load. This load should be such that symmetric and skewsymmetric forms of the deflection curve alternate alternately. Functions $X_{i}(x)$ are represented in the form of power polynomials that are easy to differentiate, integrate and calculate without the use of complex programs. When using the dynamic method, beam deflections are represented by forms of its own vibrations. In a static way, you need to build functions $X_{i}(x)$ depending on the load and reactions of the beam. In the dynamic method, it is enough to change only the values of the natural frequencies, which is very convenient.

We will keep in (2) one member of the series, which, as shown in [15], is sufficient to obtain a result of acceptable accuracy, i.e.

$$
W(x, y)=W(y) X(x) .
$$

Let's substitute (3) into (1):

$$
\begin{aligned}
& D_{1} X^{1 V} W+2 D_{3} X^{\prime \prime} W^{\prime \prime}+D_{2} X W^{1 V}+N_{x}(y) W X^{\prime \prime}+ \\
& +2 N_{x y} W^{\prime} X^{\prime}++N_{y}(x) W^{\prime \prime} X=q(x, y) .
\end{aligned}
$$

Multiply both sides of (4) by $X$ and integrate within $\left[0 ; l_{1}\right]$, where $l_{1}-$ plate dimension in axis $x$ direction (Fig. 1).

$$
\begin{aligned}
& D_{1} W \int_{0}^{l_{1}} X^{1 V} X d x+2 D_{3} W^{\prime \prime} \int_{0}^{l_{1}} X^{\prime \prime} X d x+D_{2} W^{1 V} \int_{0}^{l_{1}} X^{2} d x+ \\
& +N_{x}(y) \int_{0}^{l_{1}} X^{\prime \prime} X d x+2 W^{\prime} \int_{0}^{l_{1}} N_{x y} X^{\prime} X d x+W^{\prime \prime} \int_{0}^{l_{1}} N_{y}(x) X^{2} d x= \\
& =\int_{0}^{l_{1}} q(x, y) X d x .
\end{aligned}
$$

We introduce the notation:

$$
\begin{aligned}
& D_{2} \int_{0}^{l_{1}} X^{2} d x=A ; \int_{0}^{l_{1}}\left[2 D_{3} X^{\prime \prime}+N_{y}(x)\right] X d x=B ; \\
& \int_{0}^{l_{1}} 2 N_{x y} X^{\prime} X d x=K ; \int_{0}^{l_{1}}\left[D_{1} X^{1 V}+N_{x}(y)\right] X d x=C,
\end{aligned}
$$

then

$$
A W^{1 V}+B W^{\prime \prime}+K W^{\prime}+C W=q(y),
$$

where

$$
q(y)=\int_{0}^{l_{1}} q(x, y) X d x
$$

Coefficients $A, B, K, C$ can be calculated in any mathematical program with almost any accuracy.
From the introduced notation it follows that $N_{y}(x)$ can be any function of $x$, while $N_{x}(y)$ and $N_{y}(x)$ should be piecewise constant functions of $y$, since otherwise, equation (5) will be a differential equation with variable coefficients.

Divide all the terms of equation (5) by $A$ :

$$
W^{1 V}+2 r^{2} W^{\prime \prime}+f^{3} W^{\prime}+s^{4} W=\frac{1}{A} q(y),
$$

where

$$
r^{2}=\frac{B}{2 A} ; \quad f^{3}=\frac{K}{A} ; \quad s^{4}=\frac{C}{A}
$$

Equation (6) with initial conditions $W(0), \theta(0), M(0)$, $Q(0)$ forms the Cauchy's problem:

$$
\begin{gathered}
\left|\begin{array}{c}
W(y) \\
\theta(y) \\
M(y) \\
Q(y)
\end{array}\right|=\left|\begin{array}{cccc}
A_{11} & A_{12} & -A_{13} & -A_{14} \\
A_{21} & A_{22} & -A_{23} & -A_{13} \\
-A_{31} & -A_{32} & A_{22} & A_{12} \\
-A_{41} & -A_{31} & A_{21} & A_{11}
\end{array}\right| \begin{array}{r}
W(0) \\
\theta(0) \\
M(0) \\
Q(0)
\end{array} \mid+ \\
+\int_{0}^{y}\left|\begin{array}{c}
A_{14}(y-\xi) \\
A_{13}(y-\xi) \\
-A_{12}(y-\xi) \\
-A_{11}(y-\xi)
\end{array}\right| q(\xi) d \xi .
\end{gathered}
$$

The solution of the Cauchy problem allows us to determine the fundamental functions, the form of which depends on the roots of the characteristic equation

$$
t^{4}+2 r^{2} t^{2}+f^{3} t+s^{4}=0
$$

Consider the practically important case when $N_{x y}=0$. Moreover, in equation (7) will be $f=0$, and the roots of the characteristic equation are calculated by the formulas

$$
t_{1-4}= \pm \sqrt{r^{2} \pm \sqrt{r^{4}-s^{4}}}
$$

The form of fundamental functions is determined by the relation between $r$ and $s$, which depends on the fixing conditions of the longitudinal edges of the orthotropic plate. Four options are possible for this ratio:

1. $|s|>|r|$ - complex roots: $t_{1-4}= \pm \alpha \pm i \beta$, where $\alpha=\sqrt{\frac{s^{2}-r^{2}}{2}} ; \beta=\sqrt{\frac{s^{2}+r^{2}}{2}}$.

2. $s^{4}<0, r^{2} \neq 0 \quad-$ real and imaginary roots: $t_{1-2}= \pm \alpha ; t_{3-4}= \pm i \beta$.

3. $s^{4}>0,|s|<|r|, r^{2}>0 \quad-\quad$ imaginary roots: $t_{1-2}= \pm i \alpha ; t_{3-4}= \pm i \beta$.

4. $s^{4}>0, r^{4}-s^{4}>0, r^{2}<0-$ real and different roots: $t_{1-2}= \pm \alpha ; t_{3-4}= \pm \beta$.

After determining the fundamental functions, one can compose a transcendental equation of stability of an 
orthotropic plate, which in the general case will have the form

$$
\left|A_{*}\left(N_{x}, N_{y}, N_{x y}\right)=0\right|
$$

where $A_{*}$ - a square matrix of values of fundamental orthonormal functions with compensating elements describing the topology of the system.

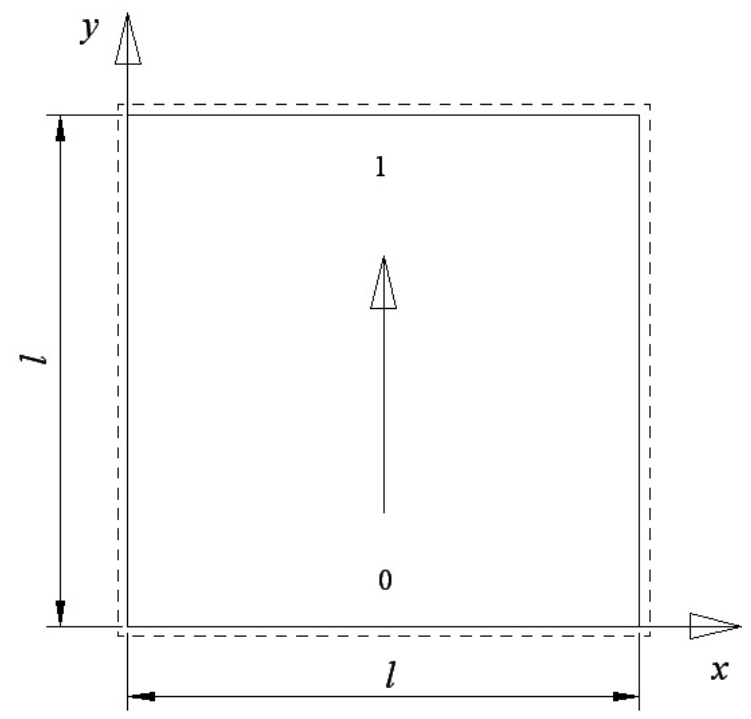

Option 1

Fig. 2. Two options of boundary conditions.

Initial data for calculation:

Elasticity moduli $-E_{x}=5 \cdot 10^{3} \mathrm{MPa}, E_{y}=200 \cdot 10^{3}$ MPa, $E_{x}=5 \cdot 10^{3} \mathrm{MPa}$; shear moduli $-G_{x y}=12,5 \cdot 10^{3}$ MPa, $G_{y x}=25 \cdot 10^{3} \mathrm{MPa}, G_{x z}=50 \cdot 10^{3} \mathrm{MPa}$; Poisson's ratios $-\mu_{x z}=0,1, \mu_{y z}=0,15, \mu_{x y}=0,0075$.

The numerical implementation of the NA BEM algorithm is performed in the Excel. The results are shown in table 1 .

Table 1. Critical loads, calculated by two methods.

\begin{tabular}{|c|c|c|c|c|c|c|}
\hline \multirow{2}{*}{$\begin{array}{c}\text { Load, } \\
\text { kN/m }\end{array}$} & \multicolumn{3}{|c|}{ Option 1 } & \multicolumn{3}{c|}{ Option 2 } \\
\hline$\sigma_{1}$ & 11395 & 11436 & 0,36 & 12320 & 12372 & 0,42 \\
\hline$\sigma_{2}$ & 11758 & 11808 & 0,42 & 35953 & 36101 & 0,41 \\
\hline$\sigma_{3}$ & 14989 & 14958 & 0,46 & 41669 & 41872 & 0,48 \\
\hline$\sigma_{4}$ & 19800 & 19902 & 0,51 & 70552 & 70855 & 0,43 \\
\hline$\sigma_{5}$ & 20002 & 20133 & 0,65 & 80201 & 80662 & 0,57 \\
\hline
\end{tabular}

To assess the accuracy of the results, the plate was modeled in ANSYS [16]. The calculation of plates by the finite element method under two variants of the boundary conditions showed good convergence of the results obtained by the two methods (Table 1). The first three forms of buckling are given in table 2 .

\section{Conclusions}

Thus, the stability problem for an orthotropic rectangular plate leads to four possible combinations of the roots of the characteristic equation of the problem, and, therefore, the complete solution of the problem will be determined
The roots of equation (8) form the spectrum of critical forces of the plate in question.

Let's look at some examples. We will determine the first three critical loads and three forms of buckling of a plate made of orthotropic material under two boundary conditions: hinged support along the entire contour (option 1) and rigid fixing of the plate on three sides with a free fourth side (option 2) (Fig. 2).

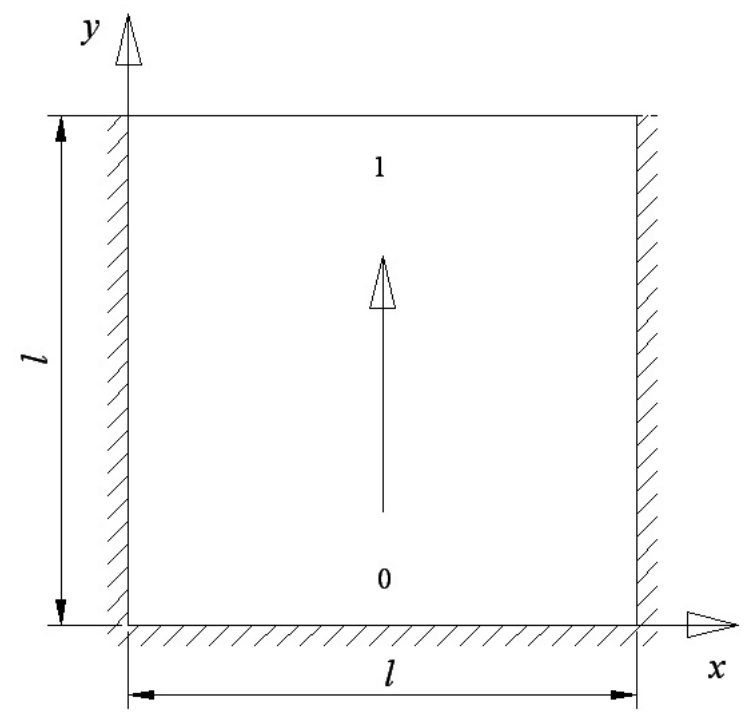

Option 2

by 64 analytical expressions of fundamental functions.

The matrix of fundamental functions, which is the basis of the transcendental stability equation, is very sparse, which significantly improves the stability of numerical operations and ensures high accuracy of the results.

An analysis of the numerical results obtained by the author's method shows very good convergence with the results of finite element analysis. For both variants of the boundary conditions, the discrepancy for the corresponding critical loads is almost the same, and increases slightly with increasing critical load.

Moreover, this discrepancy does not exceed one percent. It should be noted that for both variants of the boundary conditions, the critical loads calculated by the boundary element method are less than in the finite element calculations.

The obtained transcendental stability equation allows one to determine critical forces both by the static method and by the dynamic one. From this equation it is possible to obtain a spectrum of critical forces for a fixed number of half-waves in the direction of one of the coordinate axes. For example, one half-wave in the direction of the axis $O x$ and many half-waves in the direction of the axis $O y$ (Fig. 1), two half-waves in the direction of the axis $O x$ and many half-waves in the direction of the axis $O y$ etc., depending on the magnitude of the coefficients $A$, $B, K, C$.

The proposed approach allows us to obtain a solution to the stability problem of an orthotropic plate under any homogeneous and inhomogeneous boundary conditions. 
Table 2. Buckling forms

\begin{tabular}{|l|l|l|}
\hline Option 1 & Option 2 \\
\hline & First form \\
\hline Second form & \\
\hline
\end{tabular}

\section{References}

1. D.V. Vajnberg, E.D. Vajnberg, Plastiny, balki-stenki (Prochnost, ustojchivost i kolebaniya) (1959)

2. A.S. Volmir, Ustojchivost deformiruemyh sistem (1967)

3. S.P. Timoshenko, Ustojchivost uprugih sistem (1955)

4. S.P. Timoshenko, Ustojchivost sterzhnej, plastin $i$ obolochek (1971)

5. S.P. Timoshenko, J.M. Gere, Theory of Elastic Stability (1961)

6. S.G. Lehnickij, Teoriya uprugosti anizotropnogo tela (1977)

7. I.E. Harik, R. Ekambaram. Thin-Walled Structures 6(5), 405-416 (1988)

8. I.E. Harik, N. Balakrishnan. App. Math. Mod. 18(7), 400-402 (1994)

9. F. Bloom, D. Coffin, Handbook of Thin Plate Buckling and Postbuckling (Chapman \& Hall, 2001)

10. G.L. Kolmogorov, E.O. Zibrova, Prikl. mat. i vopr. uprav. 4, 35-42 (2015)
11. A.V. Lopatin, R.V. Avakumov, Vest. Sib. gos. aerokos. univ. im. ak. M. F. Reshetneva 28-33 (2009)

12. J. Petrolito, App. Math. Mod. 38(24), 5858-5869 (2014)

13. K.Y. Wang, P.C. Li, D.Z. Wang, Latin Am. Jour. of Sol. and Struc. 11, 2537-2554 (2014)

14. W. Jianguo, H.Maokuang. Acta Mech. Sin. 7(3), 258-266 (1991)

15. A.F. Dashenko, L.V. Kolomiec, V.F. Orobej, N.G. Surianinov, Chislenno-analiticheskij metod granichnyh elementov (2010)

16. N.N. Fedorova, S.A. Valger, M.N. Danilov, Yu.V. Zaharova, Osnovy raboty $v$ ANSYS (2017) 